Review

\title{
Efficacy of lifestyle and psychosocial interventions in reducing cognitive decline in older people: Systematic review
}

\author{
Emma Whitty $^{\mathrm{a}}$, Hassan Mansour ${ }^{\mathrm{b}}$, Elisa Aguirre ${ }^{\mathrm{a}, \mathrm{c}}$, Marina Palomo $^{\mathrm{b}, \mathrm{d}}$, Georgina Charlesworth ${ }^{\mathrm{a}, \mathrm{c}}$, \\ Serena Ramjee ${ }^{\mathrm{b}}$, Michaela Poppe ${ }^{\mathrm{b}}$, Henry Brodaty ${ }^{\mathrm{e}, \mathrm{f}}$, Helen C. Kales ${ }^{\mathrm{g}}$, Sarah Morgan-Trimmer ${ }^{\mathrm{h}}$, \\ Samuel Nyman ${ }^{\mathrm{i}}$, Iain Lang $^{\mathrm{h}}$, Kate Walters ${ }^{\mathrm{j}}$, Irene Petersen ${ }^{\mathrm{j}}$, Jennifer Wenborn ${ }^{\mathrm{a}, \mathrm{c}}$, \\ Anne -Marie Minihane ${ }^{\mathrm{k}}$, Karen Ritchie ${ }^{\mathrm{l}}$, Jonathan Huntley ${ }^{\mathrm{b}, \mathrm{d}}$, Zuzana Walker ${ }^{\mathrm{b}}$, \\ Claudia Cooper ${ }^{\mathrm{b}, \mathrm{d}, *}$
}

${ }^{a}$ UCL Department of Clinical, Educational and Health Psychology, UK

${ }^{\mathrm{b}}$ UCL Division of Psychiatry, 6th Floor Wing A Maple House, 149-150 Tottenham Court Road, London W1T 7BN, UK

${ }^{\mathrm{c}}$ North East London Mental Health Foundation Trust, UK

${ }^{\mathrm{d}}$ Camden and Islington NHS Foundation Trust, UK

${ }^{\mathrm{e}}$ Centre for Healthy Brain Ageing, University of New South Wales, Sydney, Australia

${ }^{\mathrm{f}}$ Dementia Centre for Research Collaboration, School of Psychiatry, UNSW Sydney, Australia

${ }^{\mathrm{g}}$ University California Davis, United States

${ }^{\mathrm{h}}$ University of Exeter, UK

${ }^{\mathrm{i}}$ University of Bournemouth, UK

${ }^{\mathrm{j}}$ UCL Department of Primary Care and Population Health, UK

${ }^{\mathrm{k}}$ Nutrition and Preventative Medicine, Norwich Medical School of Psychiatry, University of East Anglia, Norwich NR4 7UQ, UK

${ }^{1}$ INSERM, France

A R T I C L E I N F O

\section{Keywords:}

Dementia

Prevention

Randomised Controlled Trial

Mild Cognitive Impairment

\begin{abstract}
A B S T R A C T
It is unclear what non-pharmacological interventions to prevent cognitive decline should comprise. We systematically reviewed lifestyle and psychosocial interventions that aimed to reduce cognitive decline in healthy people aged $50+$, and people of any age with Subjective Cognitive Decline or Mild Cognitive Impairment. We narratively synthesised evidence, prioritising results from studies rated as at lower Risk of Bias (ROB) and assigning Centre for Evidence Based Medicine grades. We included 64 papers, describing: psychosocial $(n=12)$, multi-domain $(n=10)$, exercise $(n=36)$, and dietary $(n=6)$ interventions. We found Grade A evidence that over 4+ months: aerobic exercise twice weekly had a moderate effect on global cognition in people with/ without MCI; and interventions that integrate cognitive and motor challenges (e.g. dance, dumb bell training) had small to moderate effects on memory or global cognition in people with MCI. We found Grade B evidence that 4+ months of creative art or story-telling groups in people with MCI; 6 months of resistance training in people with MCI and a two-year, dietary, exercise, cognitive training and social intervention in people with or without MCI had small, positive effects on global cognition. Effects for some intervention remained up to a year beyond facilitated sessions.
\end{abstract}

\section{Introduction}

Dementia is one of the greatest health and social challenges of our time. Robust, observational study evidence indicates that it can be predicted, delayed and perhaps even prevented. A third of dementia cases have been attributable to nine risk factors (education, midlife hypertension, midlife obesity, hearing loss, late-life depression, diabetes, physical inactivity, smoking, and social isolation) (Livingston et al., 2017). These attributions do not necessarily imply that mitigation of these risk factors will prevent dementia, but recent research provides grounds for optimism. Significant reductions in UK age-standardised dementia incidence over 20 years may be attributable to increased

\footnotetext{
*Corresponding author at: UCL Division of Psychiatry, 6th Floor Wing A Maple House, 149-150 Tottenham Court Road, London W1T 7BN, UK.

E-mail addresses: e.whitty.17@ucl.ac.uk (E. Whitty), Hassan.mansour.17@ucl.ac.uk (H. Mansour), e.aguirre@ucl.ac.uk (E. Aguirre), m.palomo@ucl.ac.uk (M. Palomo), g.charlesworth@ucl.ac.uk (G. Charlesworth), serenaramjee99@gmail.com (S. Ramjee), m.poppe@ucl.ac.uk (M. Poppe), h.brodaty@unsw.edu.au (H. Brodaty), hckales@ucdavis.edu (H.C. Kales), a.minihane@uea.ac.uk (A.-M. Minihane), Claudia.cooper@ucl.ac.uk (C. Cooper).
} 
availability of education and preventive health care (Matthews et al., 2013).

There is less clear evidence from trials regarding whether interventions targeting modifiable risk factors can reduce cognitive decline or prevent dementia. Such trials have typically recruited people at increased dementia risk (older adults, or those presenting with subjective or objective cognitive impairments). Non-pharmacological approaches have sought to prevent dementia by supporting people to change their lifestyles, social activities or psychological approaches to maintaining their wellbeing (Ngandu et al., 2015). In the absence of disease-modifying treatments to prevent or delay dementia, there is hope that such approaches could significantly reduce dementia incidence, if they can be cost-effectively delivered on a large scale. There is a counter-argument, that these lifestyle factors require a societal rather than individual response (Leibing, 2018), but in the light of the burgeoning literature describing interventions designed to reduce dementia risk, we think this broad synthesis of the evidence in this area is timely.

Previous systematic reviews have evaluated specific types of interventions to reduce cognitive decline in certain populations. A recent review of longitudinal and controlled trials reported positive effects of dietary patterns including the Mediterranean, Dietary Approaches to Stop Hypertension (DASH) and anti-inflammatory diets on cognitive health outcomes in older adults (Chen et al., 2019). In a review up to 2016, voluntary weight loss in overweight/ obese people was associated with language, attention and memory improvements in seven RCTs (Veronese et al., 2017). A review of exercise interventions up to 2017 for people with MCI identified beneficial effects on global cognition of aerobic exercise programmes (D. Song et al., 2018). However, such previous reviews, which focus on single behaviours are unable to provide overall, integrated recommendations for how to reduce cognitive decline and prevent dementia in the general population.

This is to our knowledge the first comprehensive, systematic review of a broad range of lifestyle and psychosocial interventions that seek to reduce cognitive decline or prevent dementia in people at risk of dementia (due to age or cognitive frailty). We reviewed interventions that sought to act through changing lifestyle (diet or substance use, exercise), social activities and psychological approaches to life.

\section{Methods}

We registered our protocol with the PROSPERO Prospective Register of Systematic Reviews (CRD42019133614). Our research question was: How successfully have psychosocial or lifestyle interventions changed cognitive function or dementia risk in people aged $50+$, and people with subjective cognitive decline or Mild Cognitive Impairment of any age?

\subsection{Study inclusion and exclusion criteria}

We included studies reporting results from RCTs evaluating psychosocial or lifestyle interventions that aimed to reduce cognitive decline. We included a broad range of non-pharmacological interventions, where the planned mechanism of action was a change in participants' lifestyle (diet or exercise), social activities or psychological approaches. We excluded studies of computerised cognitive training interventions, as these were not considered to act through these mechanisms (and have been extensively, recently reviewed (Bhome et al., 2018; Zhang et al., 2019)). We also excluded studies that involved taking dietary supplements, which we judged to be pharmacological. We included studies of people without dementia, and where reasonable efforts had been made to exclude those with dementia (e.g. through screening with a cognitive measure using cut points validated for this purpose, or by interview). We included studies where participants were aged $50+$ (whether or not they had memory concerns); or populations of adults of any age who had some problems with memory or met criteria for mild cognitive impairment or subjective cognitive disorder but not dementia.
We included studies that compared experimental conditions to "treatment as usual", or an active control group, and excluded studies comparing two experimental conditions. We reported all objective, cognitive outcomes from identified papers, including dementia incidence.

\subsection{Search strategy}

We searched PubMed, EMBASE (Ovid) and PsycINFO (Ovid), CINAHL and Web of Science up to 30.4.19, limited to English language. We applied key terms that incorporated word combinations relating to or describing: age, study type (Randomized Controlled Trial), type of intervention (Early Medical Intervention, Therapeutics, Medical Informatics); outcome (Cognitive Dysfunction OR Dementia OR Alzheimer Disease OR mild cognitive impairment) and modifiable risk factors addressed (Diabetes Mellitus OR Exercise OR Body Mass Index, Body Weight, Smoking Cessation, Alcohol Drinking, Social Isolation, Depression, Anxiety, Cardiovascular Diseases, Vascular Diseases Blood Pressure or Hypertension). We checked references of all included papers and relevant systematic reviews, to identify any additional studies.

\subsection{Procedures}

EW screened titles and abstracts of the references identified in electronic searches, and $20 \%$ were independently checked using the pre-specified inclusion/exclusion criteria by MP. We obtained full-text copies of potentially relevant studies, and assessed them for inclusion; all were checked independently. Discrepancies were resolved by discussion, with the involvement of a third author if necessary.

\subsection{Assessing risk of bias (ROB)}

Two authors independently rated the ROB of included papers, based on responses to standard quality criteria. Each question from the quality tool checklist was rated (Yes/No). If all items asterisked below were answered affirmatively, the paper was rated as at lower ROB; we have developed this tool (modified from the Cochrane ROB tool) and used it in numerous previous reviews (Cooper et al., 2014a; Livingston et al., 2014; Lord et al., 2015; Mukadam et al., 2011; Scott et al., 2019).

1 Were participants randomised to intervention and control groups, using an independent process?*

2 Were participants and clinicians, as far as possible, masked to treatment allocation?

3 Were all participants who entered the trial accounted for and an intention-to-treat analysis conducted?*

4 Were follow-up and data collection processes the same for all participants?*

5 Was a power calculation carried out based on one of our specified outcome of interest (cognition)?

6 Were 45 (Livingston et al., 2014) or more participants included in analyses comparing treatment and control effects?*

We discussed any discrepancies in ratings and reached consensus.

\subsection{Synthesis and analysis}

We narratively synthesised findings. We calculated effect sizes where these were not available, from the mean difference, $\mathrm{n}$ and standard deviation, to facilitate interpretation of evidence. We used standard definitions of Cohen's d small (0.2), medium (0.5) and large (0.8) effect sizes. We prioritised results from studies with a lower ROB. We used Centre for Evidence Based Medicine guidelines to grade levels of evidence: Grade A (consistent evidence from studies at lower risk of bias); Grade B (consistent evidence from studies at higher risk of bias, or extrapolation from a single study at lower ROB); Grade C (extrapolations from studies at higher ROB); and Grade D (troublingly 


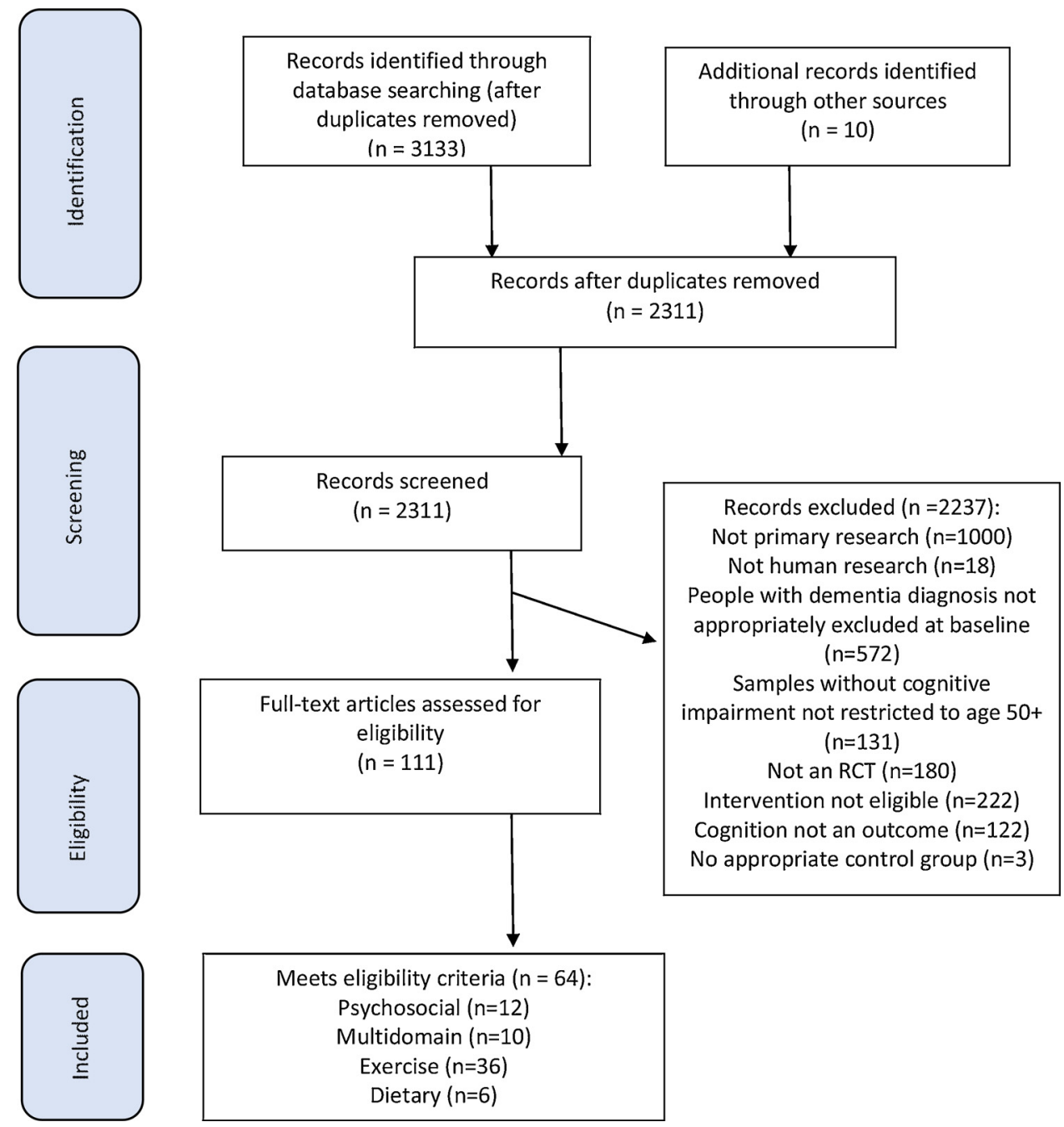

Fig. 1. PRISMA diagram of study selection.

inconsistent or inconclusive evidence at any level).

\section{Results}

Fig. 1 describes our search results. We eventually included 64 papers with 9147 participants, which took place in: Asia $(n=22)$; the USA and Canada $(n=18)$; Europe $(n=15)$, Australia $(n=6)$ and Brazil $(n=3)$. Tables $1-4$ give details of the $22(34 \%)$ studies we rated as at lower ROB, which we prioritise in our narrative synthesis. Studies rated as being at higher ROB are described in Tables 1b-41b (Supplementary tables). We evaluated results from all post-intervention outcome assessments. All but nine studies only measured outcomes immediately post-intervention. Four studies used a repeated measures designs that included immediate post-intervention and more distal outcomes in analyses (Fogarty et al., 2016; Mackin et al., 2014; Thiel et al., 2012; Zhao et al., 2018); and two studies reported immediate post-intervention and more distal end-points separately (Eyre et al., 2017; Fiatarone Singh et al., 2014). Of the studies evaluating impact after the post-intervention period, only two demonstrated evidence of effectiveness beyond the immediate post-intervention period: a year after cessation of a 6 month exercise intervention (Fiatarone Singh et al., 2014); and two months after cessation of a creative expression intervention (Zhao et al., 2018).

\subsection{Psychosocial studies}

This category included 12 studies evaluating interventions that sought to increase the amount of social contact or participation in the arts, or to change lifestyle through psychologically-focussed interventions such as goal setting, problem focussed therapies, or informational groups. All three lower ROB studies and two higher ROB studies (Wells et al., 2013; Nakatsuka et al., 2015) recruited people with MCI; one study recruited people without MCI (Dawson et al., 2014); the remainder recruited people with or without MCI.

\subsubsection{Lower risk of bias studies (Table 1)}

Two studies found that creative art groups delivered by professional therapists improved cognition in people with MCI. Zhao et al. (2018) evaluated 25 group sessions of drawing and story-telling over 16 weeks in China $(\mathrm{n}=93)$. The control group received an equivalent amount of social contact, social activation and cognitive strategy training. After six months, MoCA (Montreal Cognitive Assessment; the primary outcome) scores were significantly higher in the intervention than the control group. The small effect size was sustained for up to ten weeks postintervention.

In a smaller study, Mahendran et al. (2018) evaluated two interventions in Singapore. The first involved guided viewing and evaluation of art, narrating responses to the pieces and visual art production. The evaluation of this intervention narrowly missed the criteria for being a lower ROB study of requiring $45+$ participants in the analysis, and so 


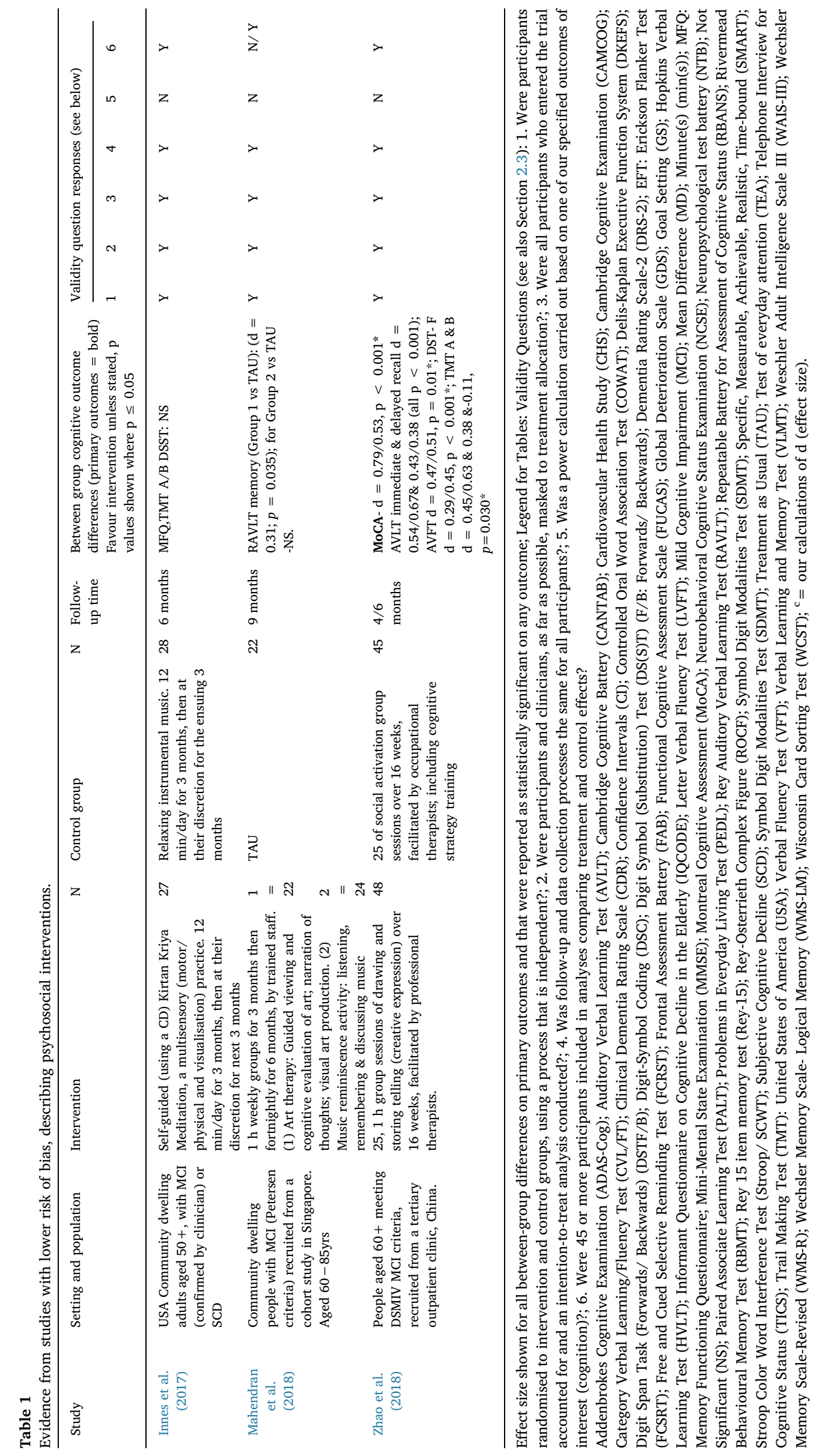


was rated as at higher ROB. It is presented here as the second comparison did meet criteria for being at lower ROB. In this second analysis, participants listened to and recalled memories associated with music. Both interventions took place in one hour, weekly groups for three months, then fortnightly for three months, led by trained facilitators. After nine months, the art therapy intervention group showed greater change in the overall memory Rey Auditory-Verbal Learning Test (RAVLT) scores compared to the "treatment as usual" group (subdomains are also reported and do not attain significance). Effect sizes were small to medium. There were no significant differences between the music reminiscence activity and control groups.

In a USA study, Innes et al. (2017) found that an individual, meditation intervention did not significantly improve cognition $(n=55)$. It was self-guided for $12 \mathrm{~min} /$ day for 3 months, then at participants' discretion for three months. Although adherence rates were high, there was no significant difference between the intervention and active control groups (who listened to instrumental music) in executive function at six months. Finally, a group intervention that sought to change lifestyle through informational groups, with or without additional counselling, did not improve global cognition relative to treatment as usual in a USA-based study $(\mathrm{n}=159)$ (Thiel et al., 2012).

\subsubsection{Higher risk of bias studies (Table 1 , online supplement)}

Only one of these studies reported significant cognitive intervention effects. This small study $(n=31)$ evaluated the impact of weekly piano lessons with home practice; executive functioning improved at 6 months, relative to treatment as usual (information was not available to calculate effect size) (Bugos et al., 2007). Of the nine other studies, three evaluated self-guided mindfulness or meditation, over 6-8 weeks; carried out individually by a research assistant (Oken et al., 2017), in an online group (Wahbeh et al., 2016), or a face to face group (Wells et al., 2013). Three studies evaluated goal or problem-focussed interventions in a variety of formats: an individual goal-setting interview for older people, with or without five follow-up mentoring phone calls, to set goals around increasing cognitive or physical activity, health, or social activity or diet (Clare et al., 2015); 3 group and 9 individual sessions including meta-cognitive strategy training for people with subjective cognitive decline (Dawson et al., 2014); and 12 individual problem-solving sessions for people with depression and executive dysfunction (Mackin et al., 2014). None reported significant effects on cognitive outcomes, though Clare et al. (2015) only intended the study as a pilot for a larger study. Finally, a home-based reminiscence intervention also reported no significant effects on cognitive outcomes (Duru Asiret and Dutkun, 2018).

\subsubsection{Summary of evidence}

- We found Grade B evidence (from one lower and one higher ROB studies) that creative art or art and story-telling groups led by trained therapists delivered over 16-26 weeks had a small effect on global cognition or memory (verbal learning) in people with MCI, relative to treatment as usual in one study and to a socially active control in the second study.

- We found Grade B evidence that self-guided meditation was ineffective at improving memory or executive functioning, relative to control conditions over 6 months (one lower ROB study) or 6-8 weeks ( 3 higher ROB studies); and that goal-focused or problemfocused interventions (setting goals to improve lifestyle, improve mood or address cognitive limitations) were ineffective at improving executive functioning or memory scores ( 3 higher ROB studies), in populations including people with and without MCI.

- We found Grade C evidence (each in one higher ROB study) that inhome reminiscence was ineffective at improving global cognition and that 6 months of weekly piano lessons was effective at improving executive functioning, in populations including people with and without MCI. 


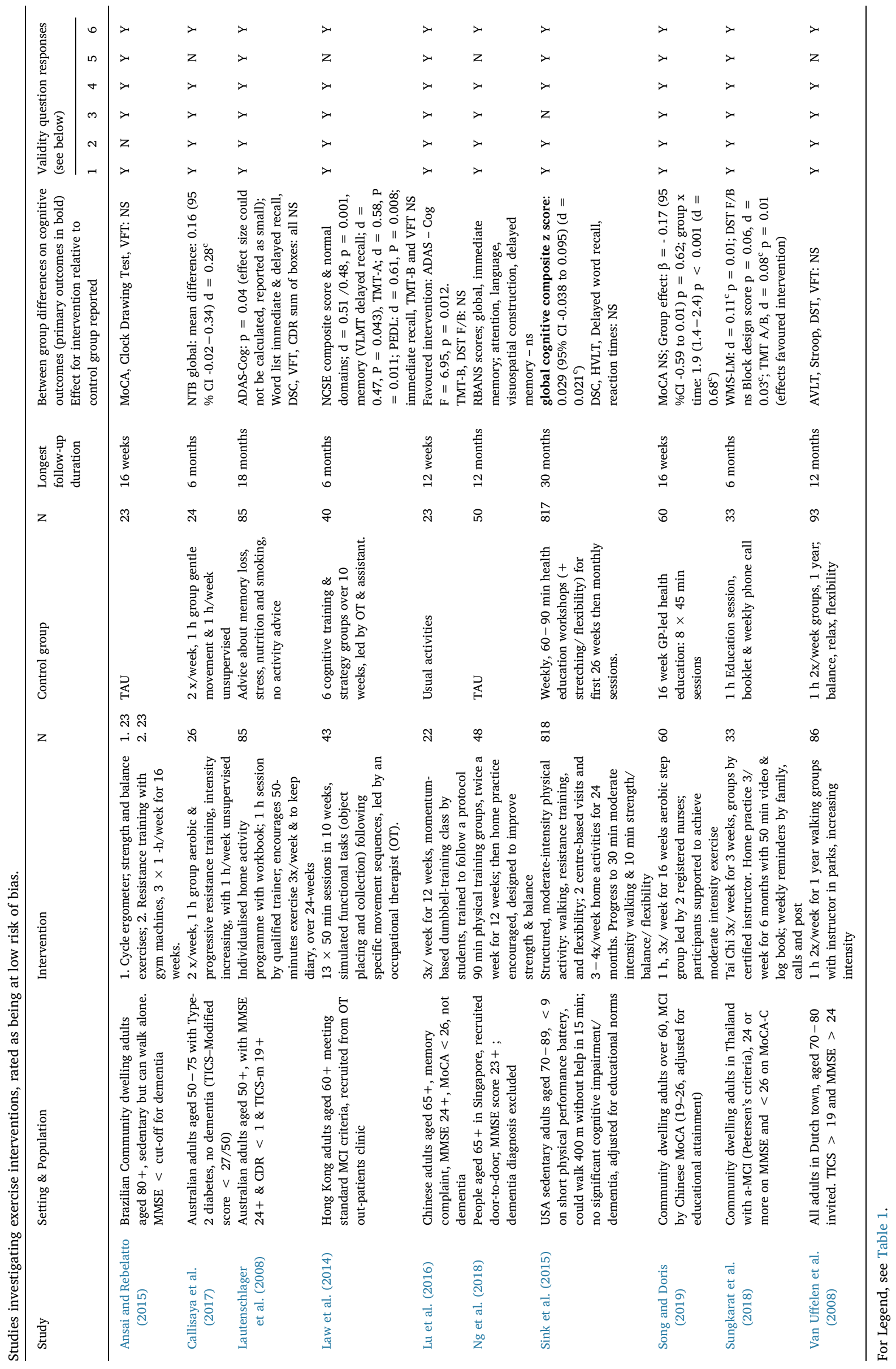




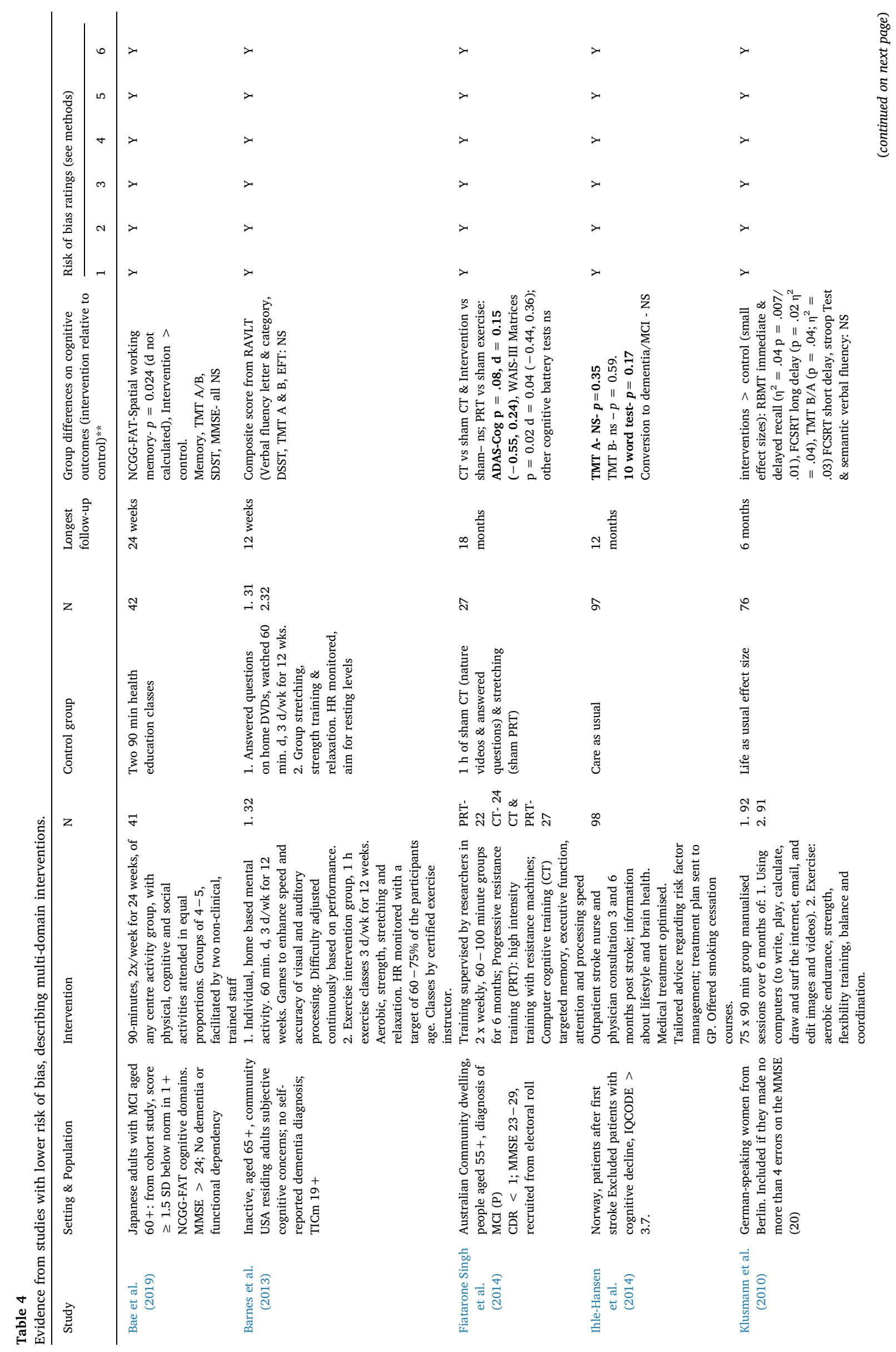




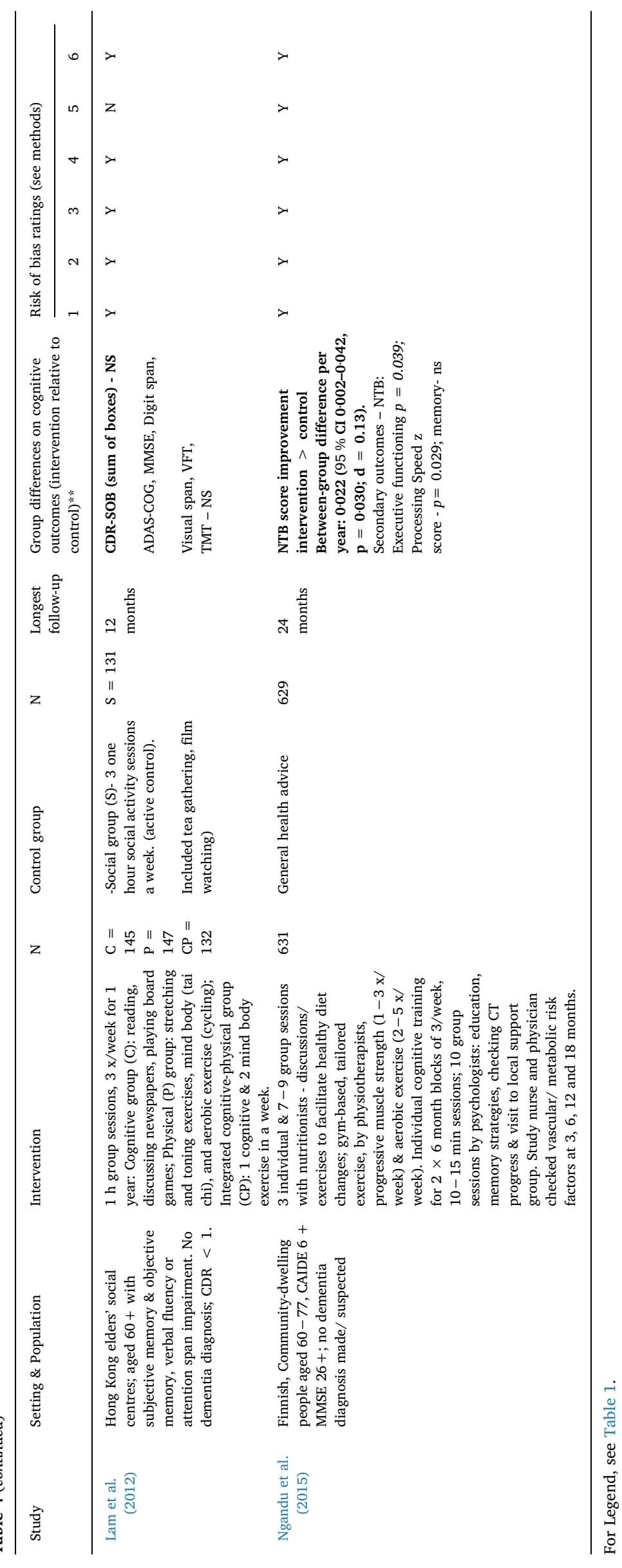




\subsection{Dietary interventions}

These six studies promoted either Mediterranean-style (plant-based) Diets (MSD), with low refined carbohydrate load, rich in fruit, vegetables and fish, and small quantities of meat, dairy and salt; or low calorie or low carbohydrate diets. Both lower ROB studies and one higher ROB study (Valls-Pedret et al., 2015) were in populations without MCI at baseline; Kwok et al. (2012) included people with and without MCI, while the remaining two studies included people with MCI only.

\subsubsection{Lower risk of bias studies (Table 2)}

Two studies compared a MSD, supported by regular (monthly or 2weekly) dietician sessions and free food, to general, low-intensity healthy eating advice. In an Australian study that recruited 166 people without MCI, there were no significant between-group differences on the global cognitive primary outcome after 6 months (Knight et al., 2016); and in the second study, in 1279 people with or without MCI, there were no significant differences between groups in global cognitive scores after one year (Marseglia et al., 2018).

\subsubsection{Higher risk of bias studies (Table $2 b$, online supplement)}

One study found that MSD, implemented through regular dietician sessions (every 6 weeks) with older people living in a residential facility and a change in facility menus, did not reduce dementia incidence over 33 months in 429 people with or without MCI (T. C. Y. Kwok et al., 2012). Horie et al. (2016) reported that group calorific restriction groups led by a nutritionist for a year were not associated with significant improvements on global or domain-specific cognitive measures in 80 people who were overweight.

Two studies reported positive findings on a proportion of cognitive outcomes evaluated. In a sub-study of the larger PREDIMED study, which unlike the larger cohort excluded people with dementia at baseline and was therefore eligible for inclusion in this review, in 340 people without MCI, memory scores improved in the Mediterranean diet plus olive oil group; and for the Mediterranean diet plus nut group (each with quarterly dietician advice) frontal and global cognitive scores improved; relative to the control group over four years, all with small effect sizes. For other cognitive scores, comparisons did not attain statistical significance, though the authors commented on the limited power of the study (Valls-Pedret et al., 2015). Finally, Krikorian et al. (2012) reported that in a small study with 23 adults with MCI, a low carbohydrate diet improved memory (with a medium effect size), but not executive function over 6 weeks, relative to a high carbohydrate diet.

\subsubsection{Summary of evidence}

- We found Grade D (inconsistent) evidence for interventions promoting MSD, with two lower (in people without MCI) and one higher ROB study (in people with or without MCI) not demonstrating effectiveness on cognitive outcomes over 6-33 months, while in one study at higher risk of bias over four years, improvements were demonstrated on global frontal and executive cognitive outcomes, with small effect sizes, in people without MCI at baseline. There was also inconsistent evidence for restriction diets from two studies involving people with MCI, with effectiveness of a calorie restriction group intervention over one year on cognition not demonstrated in people who were overweight; while six weeks of carbohydrate restriction was associated with improvements in memory (with a medium effect size) but not executive functioning.

\subsection{Exercise interventions}

We describe below evidence from populations with MCI (12 studies), without MCI (1 study), with the remaining 23 studies including both these groups.

\subsubsection{Aerobic interventions}

3.3.1.1. Lower risk of bias studies (Table 3). In Australia, an individualized home-activity program promoting $50 \mathrm{~min}$ of exercise, three times a week over 18 weeks (with $78 \%$ adherence), significantly improved global cognition, though not specific cognitive outcomes, relative to educational advice in 170 people without dementia (Lautenschlager et al., 2008). All but one participant selected aerobic exercise, most commonly walking. In a study evaluating an aerobic, step-exercise intervention compared with a health education control in 120 people with MCI, there was a moderate group-by-time effect (but not a main-group effect) on global cognition after 16 weeks; adherence rates to the intervention were $73 \%$ (Dan Song and Doris, 2019).

3.3.1.2. Higher risk of bias studies (Table 3b, supplement). A study evaluating thrice weekly aerobic groups over 12 months $(n=120)$ in Italian elders with MMSE $24+$, reported a significant, small effect on the cognitive primary outcome (MMSE) in favour of the intervention (Muscari et al., 2010). In 34 people with MCI, high-intensity aerobic exercise groups, four days a week had a significant, large effect on executive functioning (though not memory) over 6 months (Baker et al., 2010); while in a second study aerobic exercise twice a week was associated with a significant, moderate effect on global cognition over six months in 60 sedentary individuals with MCI (Langoni et al., 2019).

In contrast, a twice-weekly walking intervention (van Uffelen et al., 2008) was not associated with significant improvements in memory or executive function relative to an active control in 179 people without dementia, after a year. The authors hypothesised that low adherence (median was $63 \%$ ) might explain this, with 30 participants not attending any sessions; in women, there was an association between greater adherence and cognitive improvement in the intervention group.

\subsubsection{Resistance interventions}

3.3.2.1. Lower risk of bias studies (Table 3). In a factorial trial in people with MCI (Fiatarone Singh et al., 2014) there was a significant, small effect $(n=49)$ on the primary outcome, ADAS-Cog, immediately after the 6 month intervention, in the group receiving the resistance intervention relative to sham physical exercise (stretching) and a trend towards maintenance of this effect for one year postintervention. In two trials of shorter interventions, however, no significant effects were reported. In the first, a study evaluating a strength intervention carried out in groups for 12 weeks thrice weekly, then at home, there was little difference between the experimental and control conditions after 12 months in 98 people without dementia (adherence rates were $85 \%$ ) ( $\mathrm{Ng}$ et al., 2018). In the second ( $=69$ ), a resistance exercise protocol carried out in a gym was not associated with significant between-group differences in cognitive outcomes immediately after the 16 week intervention or 6 weeks later. Adherence was low, with only $57 \%$ of participants attending at least half of sessions (Ansai and Rebelatto, 2015).

3.3.2.2. Higher risk of bias studies (Table 3b, supplement). High-intensity resistance training once or twice a week was associated with improvements (with small effect size) on the Stroop test, the named primary outcome, but not other executive functioning tests, relative to an active control over 12 months $(n=155)$ (Liu-Ambrose et al., 2010). In a second study $(\mathrm{n}=31)$, twice-weekly resistance training under supervision over nine months was associated with better memory scores, relative to a treatment as usual control group (Busse et al., 2008).

Two resistance training interventions with a duration of 12 weeks (n $=47 ; \mathrm{n}=80$ ) (Hong et al., 2018) (Iuliano et al., 2015) were not associated with consistently significant between-group cognitive differences, though some executive functioning outcomes improved in both 
studies. An individual resistance exercise intervention $(n=43)$ was not associated with significant, cognitive effects over 4 months (Yoon et al., 2018).

\subsubsection{Multimodal exercise interventions}

3.3.3.1. Interventions that integrate motor and cognitive challenges

3.3.3.1.1. Lower risk of bias studies (Table 3). In a 13-session exercise programme over 10 weeks in which functional tasks were simulated $(\mathrm{n}=83)$, led by an occupational therapist (OT), there was a significant between-group difference on global cognitive measures at 6 months of moderate effect size that favoured the intervention, relative to usual care with cognitive strategy groups in people with MCI. This intervention combined cognitive-demanding tasks with exercise components of stretching, strengthening, balance and endurance (Law et al., 2014).

Two other interventions that combined cognitive and motor challenges were also associated with improvements in cognition. A study in 66 people with MCI reported that regular Tai Chi practice (initially with an instructor, three times a week for 3 weeks, then at home) was associated with improvements on the WMS-LM (Wechsler Memory Scale Logical memory) (primary outcome) and the Trail Making test, relative to an educational session control (with small effect sizes). The benefits of the intervention were achieved by 15 weeks and sustained for six months (Sungkarat et al., 2018). One trial that evaluated a momentumbased dumbbell-training class, thrice weekly for 12 weeks (with $84 \%$ adherence) ( $\mathrm{Lu}$ et al., 2016) in 45 people with MCI reported significantly improved global cognition and executive functioning in the intervention relative to the control group (data was not available to calculate effect size). The authors posit that variation in position changes and movement configurations during the dumbbell-training sessions involve cognitive tasks, including spatiotemporal orientation, selective attention, and executive control.

3.3.3.1.2. Higher risk of bias studies (Table $3 b$, supplement). One study reported a lack of evidence at the study end point (12 months) for a multicomponent exercise group (aerobic exercise, postural balance, and dual-task training, such as composing a poem while walking) supervised by physiotherapists, twice a week for 12 months $(\mathrm{n}=100)$ in people with MCI. Adherence to the intervention was good (80\%). However, there were significant group-by-time effects on the MMSE, and a proportion of memory and executive functioning tests, suggesting that the intervention may have been more effective over the initial six months (Suzuki et al., 2012).

Three studies evaluated dance interventions. An aerobic dance routine class thrice weekly for three months, followed by three months home practice, with additional sessions of physician counselling, was associated with a significant, small effect on the memory primary outcome, but not global cognition or executive functioning in people with or without MCI $(n=60)$ (Zhu et al., 2018). In a second study, twice weekly dance classes over 10 months were associated with improved global cognition, memory and executive functioning in people with MCI ( $\mathrm{n}=154$ ) (Lazarou et al., 2017). A dancing and handicraft group, twice a week, compared to usual activities over six months, was associated with improvements in logical memory, but not executive functioning or global cognition in people with MCI $(n=51)$ (Antunes et al., 2015).

Two studies evaluated Tai Chi in populations with MCI. Tai Chi groups held three times a week initially, then monthly for a year, compared with a stretching and toning control group reduced progression to dementia (with borderline statistical significance) $(n=389)$ (L. C. Lam et al., 2012). In a second study, a Taoist Tai Chi (TTC) group intervention (twice weekly for 10 weeks), combined with memory strategies and advice sessions, compared with the memory strategy and advice sessions alone was not associated with improvements in memory or executive functioning, over 22 weeks $(n=40$ ) (Fogarty et al., 2016).

\subsubsection{Interventions combining aerobic and strength exercises}

3.3.3.2.1. Lower risk of bias studies (Table 3). Three studies evaluated groups that combined strength and aerobic activities. In one large study $(n=1635)$ there was little difference between the experimental and control conditions after 30 months (Sink et al., 2015). In the second, small pilot study $(n=50)$, a trend favoured the intervention after 6 months (effect size was small) (Callisaya et al., 2017).

3.3.3.2.2. Higher risk of bias studies (Table $3 b$, online supplement). An aerobic and flexibility group, 3 times a week, compared to usual activities over six months, was associated with improvements on measures of logical memory, but not executive functioning or overall cognition (Antunes et al., 2015). Finally, a twice weekly aerobic and strength exercise class for 16 weeks was associated with improvements on most executive functioning measures (Vaughan et al., 2014). In single studies, a walking and social intervention (Maki et al., 2012) and an aerobic, strength and flexibility group programme carried out with an instruction video over 16 weeks (Shatil, 2013) were not associated with significant, cognitive effects.

\subsubsection{Summary of evidence}

- We found Grade A evidence (from two lower and two higher ROB studies) that aerobic exercise programmes for 16 weeks or more, at least twice a week (thrice a week in the lower ROB studies) with over $70 \%$ adherence to sessions, had a moderate sized, positive effect on global cognition, relative to control interventions in people with and without MCI.

- We found Grade A evidence (from three lower and five higher ROB study) that $1-3$ weekly group interventions lasting six months or more that combined cognitive and motor challenges (e.g. dance or dumb bell training, simulated task exercise) improved memory or global cognition by small to moderate effect sizes, in people with MCI.

- We found Grade B evidence that resistance training interventions carried out for 6 months or more, improved global functioning immediately post-intervention in people with MCI (1 lower ROB study); and in 2 higher risk of bias studies in people with or without MCI, executive functioning and memory were improved in individual resistance training studies with interventions lasting 9-12 months. Effect sizes were small.

- We found Grade B evidence (1 lower and one higher ROB study) that in people with MCI, Tai Chi sessions, initially taught by an expert and then continued at home for 15 weeks or improved global cognition, memory and executive functioning relative to stretching and toning group and educational session controls by small effect sizes, but not, in one study relative to a memory strategy group control.

- We found Grade A evidence (2 lower ROB studies and 3 higher ROB studies) that resistance training interventions lasting 4 months or less were ineffective at improving cognitive outcomes, in studies including people with or without MCI.

- We found Grade D (inconsistent) evidence (from three lower and one higher ROB study) regarding strength and aerobic groups; with one lower ROB study showing no effect and one higher ROB study showing a significant effect; with two further lower ROB studies lacking sufficient power or intervention adherence to provide a definitive result.

\subsection{Multidomain interventions}

\subsubsection{Lower risk of bias studies (Table 4)}

The FINGER (Finnish Geriatric Intervention Study to Prevent Cognitive Impairment and Disability) trial, evaluated a two-year, intensive and expert-delivered intervention including nutritional advice, physical exercise and social components, cognitive training and vascular risk management for people with a high risk for dementia score 
whose MMSE score was 26 or more. The nutrition component was based on FINNISH Dietary recommendations which like a MDS encouraged, fruit and vegetable, whole grain and fish consumption. At 24 months, Neuropsychological Test Battery score (NTB) was $25 \%$ higher in the intervention compared to a general health advice group (Ngandu et al., 2015).

Two studies reporting the impact of multi-activity group interventions, 2-3 times a week for 6 months, found beneficial effects on immediate memory outcomes, post-intervention in people with memory concerns; but inconsistent results for delayed memory and executive functioning. They evaluated thrice-weekly, group sessions on computer use and exercise classes (Klusmann et al., 2010); and twice-weekly physical, cognitive and social activities (Bae et al., 2019). Both included a control group without social stimulation (life as usual or a DVD). Another study investigated the impact of two exercise and one cognitive stimulation activity groups every week for a year and found no significant effect on global cognition or executive function (Lam et al., 2015). In this study, the active control involved attending three social activity sessions a week. In a study evaluating twice-weekly exercise, computerized cognitive training interventions and both interventions together in people with MCI, versus active control conditions, benefits of cognitive training and physical activity together on global cognition were no greater than either alone (Fiatarone Singh et al., 2014).

Other studies evaluated a home-based mental activity intervention (Barnes et al., 2013) and a clinician-delivered educational intervention providing lifestyle and brain health advice over a year to people who had experienced a first stroke (Ihle-Hansen et al., 2014). Neither was associated with significant change in cognitive outcomes.

\subsubsection{Higher risk of bias studies (Table $4 b$, online supplement)}

In one study, an intervention comprising supervised cognitive training and physical training three times a week in small groups was associated with a significant, small positive effect on ADAS-Cog (Bruno et al., 2018). In a second study, once a week memory strategies combined with lifestyle advice improved global cognition over eight weeks (T. Kwok et al., 2013). Twice weekly groups for seven weeks combining memory strategies, lifestyle factors and Cognitive Training was not associated with consistent cognitive benefits over 8 weeks (Diamond et al. (2015)).

\subsubsection{Summary of evidence from multi-domain studies}

- We found Grade B evidence (from a single lower ROB study) on a cognitive primary outcome that a two-year, intensive intervention with dietary, exercise, cognitive training and social components improved cognition with a small effect size, relative to treatment as usual, in people at risk of dementia.

- We found Grade B evidence (from a single lower ROB studies) that: 6 weeks of home-based mental activities and clinician-delivered lifestyle advice were ineffective in improving cognition.

- We found Grade D (troublingly inconsistent) evidence (from two higher ROB studies) about whether group memory strategies and lifestyle advice were effective in improving global cognition over 8 weeks.

- We found Grade D (troublingly inconsistent) evidence (from 3 lower and one higher ROB studies) for the effect of group cognitively stimulating activities combined with exercise, 2-3 times a week relative to non-socially stimulating control groups, regarding global cognition, memory and executive functioning.

\section{Discussion}

\subsection{Evidence summary}

CEBM criteria for Grade A evidence were met for the following interventions to improve global cognition or memory, when carried out over at least 4 months: aerobic exercise groups at least twice weekly, with at least $70 \%$ adherence and a moderate effect size in people with or without MCI; and 1-3 weekly groups that combined aerobic exercise with a cognitively demanding task with small to moderate effect sizes, in people with MCI. We found Grade B evidence that creative art or story-telling groups, 1-2 times a week; resistance training interventions for 6-12 months, and Tai Chi, taught by an expert and then practiced regularly at home, improved global functioning, memory or executive functioning, post-intervention, with small effect sizes in people with MCI. A two-year, intervention with dietary, exercise, cognitive training and social components also had a small, positive effect on cognition relative to treatment as usual, in people at risk of dementia. There was preliminary (Grade C) evidence that 6 months of weekly piano lessons improved executive functioning, in people with and without MCI. There was no evidence that: 4 months or less of resistance training; self-guided meditation, goal or problem-focussed interventions; or home based activities or reminiscence were effective at improving cognition.

\subsection{Interpretation of findings}

Although in many areas the evidence base is limited, there is sufficient evidence to make recommendations about interventions most likely to reduce cognitive decline in people at risk of dementia. Our results indicate that an evidence-based intervention strategy to improve global cognition, memory and executive functioning would be a group therapy carried out for 4 months or more, promoting regular (at least weekly) activity, involving aerobic or resistance exercise, with a cognitively demanding and/or creative component. A two-year intensive intervention involving dietary, exercise, cognitive training and social components (Ngandu et al., 2015) improved global cognition. Effect sizes for these interventions were mainly small, though moderate effects sizes were attained in studies evaluating aerobic exercise programmes.

The FINGER trial was an important proof-of-concept trial (Ngandu et al., 2015) but the FINGER intervention would be too expensive to implement widely. Less intensive interventions could be potentially cost-effective if they are inclusive of people from diverse socioeconomic backgrounds and minority ethnic groups who are at greatest dementia risk but currently receive least preventative care (Pham et al., 2018). The effective interventions reported here took place across the globe. There is a tension between standardizing interventions so that they adhere closely to the best available evidence, and matching advice to the activity and lifestyle preferences of the participants and the local context. For example, the two studies demonstrating effectiveness of Tai Chi took place in Thailand (Sungkarat et al., 2018) and Hong Kong (L. C. Lam et al., 2012), while a Tai Chi intervention in Canada was ineffective (Fogarty et al., 2016). The use of a potentially more active control group (memory strategies groups) or brief intervention period in the latter study may have explained this difference. It may also be that Tai Chi is more accepted in countries where it has long been a common form of exercise.

Most effective interventions involved increasing participants' activity levels at least twice a week for 6 months. To be cost-effective, future interventions will need to motivate participants to increase activity levels outside and beyond facilitated sessions. Intervention effects of aerobic exercise and resistance training were sustained for up to a year, and Tai Chi and simulated functional tasks for up to six months beyond the intervention period (Fiatarone Singh et al., 2014; Lautenschlager et al., 2008; Law et al., 2014); promoting home practice was a mechanism for this (Lautenschlager et al., 2008; Sungkarat et al., 2018).

Studies evaluating dietary interventions in isolation have not conclusively demonstrated improvements to global cognition. Epidemiological studies evaluating the impact of long term consumption of a MSD on cognitive decline indicates that it confers a $20-40 \%$ 
reduced risk of a range of disease outcome measures (DoH, 2018). The limited RCT evidence to date is largely non-supportive. However the relatively brief duration ( $<1$ year) of the dietary trials at lower ROB we evaluated might explain their ineffectiveness. For bio actives such as fish derived omega-3 fatty acids, a longer intervention period is probably needed to maximize brain concentrations (Umhau et al., 2009). Our findings accord with a systematic review published in 2018, which included five RCTs and concluded that findings were mostly non-significant, with small effect sizes. However, the significant improvements in cognitive domain composites in PREDIMED, the most robustly designed study warrant additional research (Radd-Vagenas et al., 2018).

\subsection{Limitations}

Most studies did not identify a primary outcome. Lack of evidence of effectiveness is not proof of lack of effectiveness; there is insufficient evidence to guide content and duration of future interventions with greater specificity that we provide here. We identify several areas where the evidence base is currently troublingly inconsistent. The populations of included studies may not be generalisable; men, people from white ethnic backgrounds and younger people are over-represented in dementia research (Cooper et al., 2014b). Only two lower ROB studies measured impact on dementia incidence (Lam et al., 2012; Lautenschlager et al., 2008) and neither reported a significant effect, probably reflecting the very large sample size and/or long duration of follow-up required to have power to detect a significant finding on this outcome. Some of the included studies delineated a specific population, such as people with MCI, but many included any person within age limits who did not have dementia at baseline (most likely encompassing healthy older adults and people with Subjective Cognitive Decline and MCI). Such pragmatism reflects how dementia prevention interventions are likely to be implemented if taken up by public health programmes, but there are issues with combining these groups in analyses, as they are likely to differ in their ability and motivation to engage. We decided to undertake a narrative review, to capture the variety of population, interventions, follow-up durations and outcomes these studies have reported. We have distinguished between studies that recruited people with MCI, people without MCI, or both. This is an important distinction as psychosocial dysfunction is associated with cognitive decline (Lozupone et al., 2018). No study recruited people specifically with subjective cognitive decline. We describe and consider adherence rates to interventions where they are reported; we note, for example that aerobic exercise interventions were only effective where $70 \%$ or more adherence was reported. Not all interventions were fully specified or standardised; for example, for 5 studies, the intervention duration was not reported. We considered that a meta-analysis would be less informative in the light of this heterogeneity.

\subsection{Conclusions}

Based on current published evidence, an evidence-based intervention strategy to improve global cognition, memory and executive functioning would be a group therapy carried out for 4 months or more, promoting engagement in regular (at least weekly) activity, involving aerobic or resistance exercise, and cognitively demanding (visuospatial/ memory) or creative tasks. There is some evidence that intervention effects may be sustained for up to a year beyond the initial intervention period; this is promising and may be critical to the development of effective and cost-effective interventions.

\section{Funding}

This work was supported by the ESRC/NIHR [ES/S010408/1].

\section{Declaration of Competing Interest}

None.

\section{Appendix A. Supplementary data}

Supplementary material related to this article can be found, in the online version, at doi:https://doi.org/10.1016/j.arr.2020.101113.

\section{References}

Ansai, J.H., Rebelatto, J.R., 2015. Effect of two physical exercise protocols on cognition and depressive symptoms in oldest-old people: a randomized controlled trial. Geriatr. Gerontol. Int. 15 (9), 1127-1134. https://doi.org/10.1111/ggi.12411.

Antunes, H.K., Santos-Galduroz, R.F., De Aquino Lemos, V., Bueno, O.F., Rzezak, P., de Santana, M.G., De Mello, M.T., 2015. The influence of physical exercise and leisure activity on neuropsychological functioning in older adults. Age (Dordr) 37 (4), 9815. https://doi.org/10.1007/s11357-015-9815-8.

Bae, S., Lee, S., Lee, S., Jung, S., Makino, K., Harada, K., et al., 2019. The effect of a multicomponent intervention to promote community activity on cognitive function in older adults with mild cognitive impairment: a randomized controlled trial. Complement. Ther. Med. 42, 164-169. https://doi.org/10.1016/j.ctim.2018.11.011.

Baker, L.D., Frank, L.L., Foster-Schubert, K., Green, P.S., Wilkinson, C.W., McTiernan, A., et al., 2010. Aerobic exercise improves cognition for older adults with glucose intolerance, a risk factor for Alzheimer's disease. J. Alzheimers Dis. 22 (2), 569-579. https://doi.org/10.3233/jad-2010-100768.

Barnes, D.E., Santos-Modesitt, W., Poelke, G., Kramer, A.F., Castro, C., Middleton, L.E., Yaffe, K., 2013. The mental activity and eXercise (MAX) trial a randomized controlled trial to enhance cognitive function in older adults. JAMA Intern. Med. 173 (9), 797-804. https://doi.org/10.1001/jamainternmed.2013.189.

Bhome, R., Berry, A.J., Huntley, J.D., Howard, R.J., 2018. Interventions for subjective cognitive decline: systematic review and meta-analysis. BMJ Open 8 (7), e021610. https://doi.org/10.1136/bmjopen-2018-021610.

Bruno, R.M., Stea, F., Sicari, R., Ghiadoni, L., Taddei, S., Ungar, A., et al., 2018. Vascular function is improved after an environmental enrichment program: the train the brainmind the vessel study. Hypertension 71 (6), 1218-1225. Retrieved from. http:// ovidsp.ovid.com/ovidweb.cgi? T $=$ JS\&CSC $=$ Y\&NEWS $=$ N\&PAGE $=$ fulltext\&D $=$ medl $\& \mathrm{AN}=29632101$

Bugos, J.A., Perlstein, W.M., McCrae, C.S., Brophy, T.S., Bedenbaugh, P.H., 2007. Individualized piano instruction enhances executive functioning and working memory in older adults. Aging Ment. Health 11 (4), 464-471.

Busse, A.L.F., WJ, Magaldi, R.M., Coelho, V.A., Melo, A.C., Betoni, R.A., Sanarem, K.M., 2008. Effects of resistance training exercise on cognitive performance in elderly individuals with memory impairment: results of a controlled trial. Einstein 6, 5 .

Callisaya, M.L., Daly, R.M., Sharman, J.E., Bruce, D., Davis, T.M.E., Greenaway, T., et al., 2017. Feasibility of a multi-modal exercise program on cognition in older adults with Type 2 diabetes - a pilot randomised controlled trial. BMC Geriatr. 17 (1), 237. https://doi.org/10.1186/s12877-017-0635-9.

Chen, X., Maguire, B., Brodaty, H., O'Leary, F., 2019. Dietary patterns and cognitive health in older adults: a systematic review. J. Alzheimers Dis. 67 (2), 583-619. https://doi.org/10.3233/JAD-180468.

Clare, L., Nelis, S.M., Jones, I.R., Hindle, J.V., Thom, J.M., Nixon, J.A., et al., 2015. The Agewell trial: a pilot randomised controlled trial of a behaviour change intervention to promote healthy ageing and reduce risk of dementia in later life. BMC Psychiatry 15. https://doi.org/10.1186/s12888-015-0402-4.

Cooper, C., Ketley, D., Livingston, G., 2014a. Systematic review and meta-analysis to estimate potential recruitment to dementia intervention studies. Int. J. Geriatr. Psychiatry 29 (5), 515-525. https://doi.org/10.1002/gps.4034.

Cooper, C., Ketley, D., Livingston, G., 2014b. Systematic review and meta-analysis to estimate potential recruitment to dementia intervention studies. Int. J. Geriatr. Psychiatry 29 (5), 515-525.

Dawson, D., Richardson, J., Troyer, A., Binns, M., Clark, A., Polatajko, H., et al., 2014. An occupation-based strategy training approach to managing age-related executive changes: a pilot randomized controlled trial. Clin. Rehabil. 28 (2), 118-127. https:// doi.org/10.1177/0269215513492541.

Diamond, K., Mowszowski, L., Cockayne, N., Norrie, L., Paradise, M., Hermens, D.F., et al., 2015. Randomized controlled trial of a healthy brain ageing cognitive training program: effects on memory, mood, and sleep. J. Alzheimer Dis. 44 (4), 1181-1191. https://doi.org/10.3233/jad-142061.

DoH, 2018. SACN Statement on Diet, Cognitive Impairment and Dementia.

Duru Asiret, G., Dutkun, M., 2018. The effect of reminiscence therapy on the adaptation of elderly women to old age: a randomized clinical trial. Complement. Ther. Med. 41, 124-129. https://doi.org/10.1016/j.ctim.2018.09.018.

Eyre, H.A., Siddarth, P., Acevedo, B., Van Dyk, K., Paholpak, P., Ercoli, L., et al., 2017. A randomized controlled trial of Kundalini yoga in mild cognitive impairment. Int. Psychogeriatr. 29 (4), 557-567. https://doi.org/10.1017/S1041610216002155.

Fiatarone Singh, M.A., Gates, N., Saigal, N., Wilson, G.C., Meiklejohn, J., Brodaty, H., et al., 2014. The Study of Mental and Resistance Training (SMART) study-resistance training and/or cognitive training in mild cognitive impairment: a randomized, double-blind, double-sham controlled trial. J. Am. Med. Dir. Assoc. 15 (12), 873-880. https://doi.org/10.1016/j.jamda.2014.09.010.

Fogarty, J.N., Murphy, K.J., McFarlane, B., Montero-Odasso, M., Wells, J., Troyer, A.K., 
et al., 2016. Taoist Tai Chi(R) and memory intervention for individuals with mild cognitive impairment. J. Aging Phys. Act. 24 (2), 169-180. https://doi.org/10.1123/ japa.2014-0062.

Hong, S.G., Kim, J.H., Jun, T.W., 2018. Effects of 12-Week resistance exercise on electroencephalogram patterns and cognitive function in the elderly with mild cognitive impairment: a randomized controlled trial. Clin. J. Sport Med. 28 (6), 500-508. https://doi.org/10.1097/jsm.0000000000000476.

Horie, N.C., Serrao, V.T., Simon, S.S., Gascon, M.R., Dos Santos, A.X., Zambone, M.A., et al., 2016. Cognitive effects of intentional weight loss in elderly obese individuals with mild cognitive impairment. J. Clin. Endocrinol. Metab. 101 (3), 1104-1112. https://doi.org/10.1210/jc.2015-2315.

Ihle-Hansen, H., Thommessen, B., Fagerland, M.W., Øksengård, A.R., Wyller, T.B. Engedal, K., Fure, B., 2014. Multifactorial vascular risk factor intervention to prevent cognitive impairment after stroke and TIA: a 12-month randomized controlled trial. Int. J. Stroke 9 (7), 932-938

Innes, K.E., Selfe, T.K., Khalsa, D.S., Kandati, S., 2017. Meditation and music improve memory and cognitive function in adults with subjective cognitive decline: a pilot randomized controlled trial. J. Alzheimer Dis. 56 (3), 899-916. https://doi.org/10 3233/jad-160867.

Iuliano, E., di Cagno, A., Aquino, G., Fiorilli, G., Mignogna, P., Calcagno, G., Di Costanzo, A., 2015. Effects of different types of physical activity on the cognitive functions and attention in older people: a randomized controlled study. Exp. Gerontol. 70, 105-110. https://doi.org/10.1016/j.exger.2015.07.008.

Klusmann, V., Evers, A., Schwarzer, R., Schlattmann, P., Reischies, F.M., Heuser, I., Dimeo, F.C., 2010. Complex mental and physical activity in older women and cognitive performance: a 6-month randomized controlled trial. J. Gerontol. A Biol. Sci. Med. Sci. 65 (6), 680-688. https://doi.org/10.1093/gerona/glq053.

Knight, A., Bryan, J., Wilson, C., Hodgson, J.M., Davis, C.R., Murphy, K.J., 2016. The mediterranean diet and cognitive function among healthy older adults in a 6-Month randomised controlled trial: the MedLey study. Nutrients 8 (9). https://doi.org/10. 3390/nu8090579.

Krikorian, R., Shidler, M.D., Dangelo, K., Couch, S.C., Benoit, S.C., Clegg, D.J., 2012. Dietary ketosis enhances memory in mild cognitive impairment. Neurobiol. Aging 33 (2), e19-e27 Retrieved fromhttp://ovidsp.ovid.com/ovidweb.cgi? $\mathrm{T}=\mathrm{JS} \& \mathrm{CSC}=\mathrm{Y} \&$ NEWS $=$ N\&PAGE $=$ fulltext $\& D=$ psyc9\&AN $=2011-28364-045$ https $: / /$ ucl-new-primo.hosted.exlibrisgroup.com/openurl/UCL/UCL_VU2?sid= OVID:psycdb\&id = pmid: \&id = doi:10.1016\%2Fj.neurobiolaging.2010.10.006\&issn $=0197-4580 \& i s b n=\&$ vo lume $=33 \&$ issue $=2 \&$ spage $=$ e19\&pages $=$ e19-e27\&date $=2012 \&$ title $=$ Neurobiology + of + Aging\&atitle $=$ Dietary + ketosis + enhances + memory + in + mild + cognitive + impairment.\&aulast $=$ Krikorian

Kwok, T.C.Y., Lam, L.C.W., Sea, M.M.M., Goggins, W., Woo, J., 2012. A randomized controlled trial of dietetic interventions to prevent cognitive decline in old age hostel residents. Eur. J. Clin. Nutr. 66 (10), 1135-1140. https://doi.org/10.1038/ejcn. 2012.117.

Kwok, T., Wong, A., Chan, G., Shiu, Y.Y., Lam, K.C., Young, D., et al., 2013. Effectiveness of cognitive training for Chinese elderly in Hong Kong. Clin. Interv. Aging 8, 213-219. https://doi.org/10.2147/cia.s38070.

Lam, L.C., Chau, R.C., Wong, B.M., Fung, A.W., Tam, C.W., Leung, G.T., et al., 2012. A 1year randomized controlled trial comparing mind body exercise (Tai Chi) with stretching and toning exercise on cognitive function in older Chinese adults at risk of cognitive decline. J. Am. Med. Dir. Assoc. 13 (6), e515-520. https://doi.org/10. 1016/j.jamda.2012.03.008. 568

Lam, L.C.W., Chan, W.C., Leung, T., Fung, A.W.T., Leung, E.M.F., 2015. Would older adults with mild cognitive impairment adhere to and benefit from a structured lifestyle activity intervention to enhance cognition?: a cluster randomized controlled trial. PLoS One 10 (3). https://doi.org/10.1371/journal.pone.0118173.

Langoni, C.D.S., Resende, T.L., Barcellos, A.B., Cecchele, B., Knob, M.S., Silva, T.D.N., et al., 2019. Effect of exercise on cognition, conditioning, muscle endurance, and balance in older adults with mild cognitive impairment: a randomized controlled trial. J. Geriatr. Phys. Ther. 42 (2), E15-E22. https://doi.org/10.1519/JPT. 0000000000000191.

Lautenschlager, N.T., Cox, K.L., Flicker, L., Foster, J.K., van Bockxmeer, F.M., Xiao, J. et al., 2008. Effect of physical activity on cognitive function in older adults at risk for Alzheimer disease: a randomized trial. Jama 300 (9), 1027-1037.

Law, L.L., Barnett, F., Yau, M.K., Gray, M.A., 2014. Effects of functional tasks exercise on older adults with cognitive impairment at risk of Alzheimer's disease: a randomised controlled trial. Age Ageing 43 (6), 813-820.

Lazarou, I., Parastatidis, T., Tsolaki, A., Gkioka, M., Karakostas, A., Douka, S., Tsolaki, M., 2017. International ballroom dancing against neurodegeneration: a randomized controlled trial in Greek community-dwelling elders with mild cognitive impairment. Am. J. Alzheimers Dis. Other Demen. 32 (8), 489-499. https://doi.org/10.1177/ 1533317517725813.

Leibing, A., 2018. Situated prevention: framing the "new dementia". J. Law Med. Ethics 46 (3), 704-716. https://doi.org/10.1177/1073110518804232.

Liu-Ambrose, T., Nagamatsu, L.S., Graf, P., Beattie, B.L., Ashe, M.C., Handy, T.C., 2010. Resistance training and executive functions: a 12-month randomized controlled trial. Arch. Intern. Med. 170 (2), 170-178. https://doi.org/10.1001/archinternmed.2009. 494.

Livingston, G., Kelly, L., Lewis-Holmes, E., Baio, G., Morris, S., Patel, N., et al., 2014. A systematic review of the clinical effectiveness and cost-effectiveness of sensory, psychological and behavioural interventions for managing agitation in older adults with dementia. Health Technol.Assess. 18 (39), 1-vi. https://doi.org/10.3310/ hta18390.

Livingston, G., Sommerlad, A., Orgeta, V., Costafreda, S., G, Huntley, J., Ames, D., et al., 2017. Dementia prevention, intervention, and care. Lancet 390 (10113), 2673-2734. https://doi.org/10.1016/S0140-6736(17)31363-6.
Lord, K., Livingston, G., Cooper, C., 2015. A systematic review of barriers and facilitators to and interventions for proxy decision-making by family carers of people with dementia. Int. Psychogeriatr. 27 (8), 1301-1312. https://doi.org/10.1017/ s1041610215000411.

Lozupone, M., Panza, F., Piccininni, M., Copetti, M., Sardone, R., Imbimbo, B.P., et al., 2018. Social dysfunction in older age and relationships with cognition, depression, and apathy: the GreatAGE study. J. Alzheimers Dis. 65 (3), 989-1000. https://doi. org/10.3233/JAD-180466.

Lu, J., Sun, M., Liang, L., Feng, Y., Pan, X., Liu, Y., 2016. Effects of momentum-based dumbbell training on cognitive function in older adults with mild cognitive impairment: a pilot randomized controlled trial. Clin. Interv. Aging 11, 9-16. https://doi. org/10.2147/CIA.S96042.

Mackin, R.S., Nelson, J.C., Delucchi, K., Raue, P., Byers, A., Barnes, D., et al., 2014. Cognitive outcomes after psychotherapeutic interventions for major depression in older adults with executive dysfunction. Am. J. Geriatr. Psychiatry 22 (12), 1496-1503. https://doi.org/10.1016/j.jagp.2013.11.002.

Mahendran, R., Gandhi, M., Moorakonda, R.B., Wong, J., Kanchi, M.M., Fam, J., et al., 2018. Art therapy is associated with sustained improvement in cognitive function in the elderly with mild neurocognitive disorder: findings from a pilot randomized controlled trial for art therapy and music reminiscence activity versus usual care. Trials 19. https://doi.org/10.1186/s13063-018-2988-6.

Maki, Y., Ura, C., Yamaguchi, T., Murai, T., Isahai, M., Kaiho, A., et al., 2012. Effects of intervention using a community-based walking program for prevention of mental decline: a randomized controlled trial. J. Am. Geriatr. Soc. 60 (3), 505-510.

Marseglia, A., Xu, W.L., Fratiglioni, L., Fabbri, C., Berendsen, A.A.M., Bialecka-Debek, A., et al., 2018. Effect of the NU-AGE diet on cognitive functioning in older adults: a randomized controlled trial. Front. Physiol. 1. https://doi.org/10.3389/fphys.2018. 00349.

Matthews, F.E., Arthur, A., Barnes, L.E., Bond, J., Jagger, C., Robinson, L., Brayne, C., 2013. A two-decade comparison of prevalence of dementia in individuals aged 65 years and older from three geographical areas of England: results of the Cognitive Function and Ageing Study I and II. Lancet 382 (9902), 1405-1412 Retrieved from $<$ Go to ISI $>: / / 000326237500025$.

Mukadam, N., Cooper, C., Livingston, G., 2011. A systematic review of ethnicity and pathways to care in dementia. Int. J. Geriatr. Psychiatry 26 (1), 12-20. https://doi. org/10.1002/gps. 2484.

Muscari, A., Giannoni, C., Pierpaoli, L., Berzigotti, A., Maietta, P., Foschi, E., et al., 2010. Chronic endurance exercise training prevents aging-related cognitive decline in healthy older adults: a randomized controlled trial. Int. J. Geriatr. Psychiatry 25 (10), 1055-1064. https://doi.org/10.1002/gps.2462.

Ng, T.P., Ling, L.H.A., Feng, L., Nyunt, M.S.Z., Feng, L., Niti, M., et al., 2018. Cognitive effects of multi-domain interventions among pre-frail and frail community-living older persons: randomized controlled trial. J. Gerontol. A Biol. Sci. Med. Sci. 73 (6), 806-812. https://doi.org/10.1093/gerona/glx207.

Ngandu, T., Lehtisalo, J., Solomon, A., Levalahti, E., Ahtiluoto, S., Antikainen, R., et al., 2015. A 2 year multidomain intervention of diet, exercise, cognitive training, and vascular risk monitoring versus control to prevent cognitive decline in at-risk elderly people (FINGER): a randomised controlled trial. Lancet 385 (9984), 2255-2263. https://doi.org/10.1016/S0140-6736(15)60461-60465 [doi]. S0140-6736(15) 60461-60465 [pii]

Oken, B.S., Wahbeh, H., Goodrich, E., Klee, D., Memmott, T., Miller, M., Fu, R.W., 2017. Meditation in stressed older adults: improvements in self-rated mental health not paralleled by improvements in cognitive function or physiological measures. Mindfulness 8 (3), 627-638. https://doi.org/10.1007/s12671-016-0640-7.

Pham, T.M., Petersen, I., Walters, K., Raine, R., Manthorpe, J., Mukadam, N., Cooper, C., 2018. Trends in dementia diagnosis rates in UK ethnic groups: analysis of UK primary care data. Clin. Epidemiol. 10, 949-960. https://doi.org/10.2147/CLEP.S152647.

Radd-Vagenas, S., Duffy, S.L., Naismith, S.L., Brew, B.J., Flood, V.M., Fiatarone Singh, M.A., 2018. Effect of the Mediterranean diet on cognition and brain morphology and function: a systematic review of randomized controlled trials. Am. J. Clin. Nutr. 107 (3), 389-404. https://doi.org/10.1093/ajcn/nqx070.

Scott, I., Cooper, C., Leverton, M., Burton, A., Beresford-Dent, J., Rockwood, K., et al., 2019. Effects of nonpharmacological interventions on functioning of people living with dementia at home: a systematic review of randomised controlled trials. Int. J. Geriatr. Psychiatry 34 (10), 1386-1402. https://doi.org/10.1002/gps.5127.

Shatil, E., 2013. Does combined cognitive training and physical activity training enhance cognitive abilities more than either alone? A four-condition randomized controlled trial among healthy older adults. Front. Aging Neurosci. 5, 8. https://doi.org/10. 3389/fnagi.2013.00008.

Sink, K.M., Espeland, M.A., Castro, C.M., Church, T., Cohen, R., Dodson, J.A., et al., 2015. Effect of a 24-Month physical activity intervention vs health education on cognitive outcomes in sedentary older adults: the LIFE randomized trial. JAMA 314 (8), 781-790 Retrieved fromhttp://ovidsp.ovid.com/ovidweb.cgi? $\mathrm{T}=\mathrm{JS} \& \mathrm{CSC}=\mathrm{Y} \&$ NEWS $=\mathrm{N} \& \mathrm{PAGE}=$ fulltext $\& \mathrm{D}=$ med11\&AN $=26305648 \mathrm{https}: / /$ jamanetwork.com $/$ journals/jama/articlepdf/2429712/joi150091.pdf.

Song, D., Doris, S., 2019. Effects of a moderate-intensity aerobic exercise programme on the cognitive function and quality of life of community-dwelling elderly people with mild cognitive impairment: a randomised controlled trial. Int. J. Nurs. Stud. 93 97-105.

Song, D., Yu, D.S.F., Li, P.W.C., Lei, Y., 2018. The effectiveness of physical exercise on cognitive and psychological outcomes in individuals with mild cognitive impairment: a systematic review and meta-analysis. Int. J. Nurs. Stud. 79, 155-164. https://doi. org/10.1016/j.ijnurstu.2018.01.002.

Sungkarat, S., Boripuntakul, S., Kumfu, S., Lord, S.R., Chattipakorn, N., 2018. Tai Chi improves cognition and plasma BDNF in older adults with mild cognitive impairment: a randomized controlled trial. Neurorehabil. Neural Repair 32 (2), 142-149. 
Suzuki, T., Shimada, H., Makizako, H., Doi, T., Yoshida, D., Tsutsumimoto, K., et al., 2012. Effects of multicomponent exercise on cognitive function in older adults with amnestic mild cognitive impairment: a randomized controlled trial. BMC Neurol. 12, 128. https://doi.org/10.1186/1471-2377-12-128.

Thiel, C., Vogt, L., Tesky, V.A., Meroth, L., Jakob, M., Sahlender, S., et al., 2012. Cognitive intervention response is related to habitual physical activity in older adults. Aging Clin. Exp. Res. 24 (1), 47-55. https://doi.org/10.3275/7569.

Umhau, J.C., Zhou, W., Carson, R.E., Rapoport, S.I., Polozova, A., Demar, J., et al., 2009. Imaging incorporation of circulating docosahexaenoic acid into the human brain using positron emission tomography. J. Lipid Res. 50 (7), 1259-1268. https://doi. org/10.1194/jlr.M800530-JLR200.

Valls-Pedret, C., Sala-Vila, A., Serra-Mir, M., Corella, D., de la Torre, R., MartinezGonzalez, M.A., et al., 2015. Mediterranean diet and age-related cognitive decline a randomized clinical trial. JAMA Intern. Med. 175 (7), 1094-1103. https://doi.org/ 10.1001/jamainternmed.2015.1668.

van Uffelen, J.G., Chinapaw, M.J., van Mechelen, W., Hopman-Rock, M., 2008. Walking or vitamin B for cognition in older adults with mild cognitive impairment? A randomised controlled trial. Br. J. Sports Med. 42 (5), 344-351. https://doi.org/10. 1136/bjsm.2007.044735.

Vaughan, S., Wallis, M., Polit, D., Steele, M., Shum, D., Morris, N., 2014. The effects of multimodal exercise on cognitive and physical functioning and brain-derived neurotrophic factor in older women: a randomised controlled trial. Age Ageing 43 (5), 623-629. https://doi.org/10.1093/ageing/afu010.

Veronese, N., Facchini, S., Stubbs, B., Luchini, C., Solmi, M., Manzato, E., et al., 2017.
Weight loss is associated with improvements in cognitive function among overweight and obese people: a systematic review and meta-analysis. Neurosci. Biobehav. Rev. 72, 87-94. https://doi.org/10.1016/j.neubiorev.2016.11.017.

Wahbeh, H., Goodrich, E., Oken, B.S., 2016. Internet-based mindfulness meditation for cognition and mood in older adults: a pilot study. Altern. Ther. Health Med. 22 (2), 44-53. Retrieved from ://WOS:000384938000007. https://www.ncbi.nlm.nih.gov/ pmc/articles/PMC4874472/pdf/nihms784909.pdf.

Wells, R.E., Kerr, C.E., Wolkin, J., Dossett, M., Davis, R.B., Walsh, J., et al., 2013. Meditation for adults with mild cognitive impairment: a pilot randomized trial. J. Am. Geriatr. Soc. 61 (4), 642-645. https://doi.org/10.1111/jgs.12179.

Yoon, D.H., Lee, J.Y., Song, W., 2018. Effects of resistance exercise training on cognitive function and physical performance in cognitive frailty: a randomized controlled trial. J. Nutr. Health Aging 22 (8), 944-951. https://doi.org/10.1007/s12603-018-1090-9.

Zhang, H., Huntley, J., Bhome, R., Holmes, B., Cahill, J., Gould, R.L., et al., 2019. Effect of computerised cognitive training on cognitive outcomes in mild cognitive impairment: a systematic review and meta-analysis. BMJ Open 9 (8), e027062. https://doi.org/ 10.1136/bmjopen-2018-027062.

Zhao, J.Y., Li, H., Lin, R., Wei, Y., Yang, A.P., 2018. Effects of creative expression therapy for older adults with mild cognitive impairment at risk of Alzheimer's disease: a randomized controlled clinical trial. Clin. Interv. Aging 13, 1313-1320. https://doi org/10.2147/cia.s161861.

Zhu, Y., Wu, H., Qi, M., Wang, S., Zhang, Q., Zhou, L., et al., 2018. Effects of a specially designed aerobic dance routine on mild cognitive impairment. Clin. Interv. Aging 13, 1691-1700. https://doi.org/10.2147/cia.s163067. 